\title{
Sterhl ratio of vortex beams propagating through atmospheric turbulence
}

\author{
Qiangbo Suo, Jinhong Li ,Linying Chen \\ Department of Physics, Taiyuan University of Science and Technology, Taiyuan 030024, China \\ e-mail:690374182@qq.com, jinhongli@live.cn(Corresponding author),cly000818@sohu.com
}

Keywords: Sterhl ratio; atmospheric turbulence; vortex beam

\begin{abstract}
Based on the extended Huygens-Fresnel principle, the analytical expressions for the Strehl ratio( $\left.\mathrm{S}_{\mathrm{R}}\right)$ of Gaussian Schell-model(GSM) vortex beams and Gaussian Schell-model non-vortex beams propagating through atmospheric turbulence and free space are derived. The corresponding results are also calculated. It is shown that the smaller the structure constant $C_{\mathrm{n}}^{2}$, the inner scale $I_{0}$ and the wave length $\lambda$, as well as the larger the outer scale turbulence $L_{0}$, the lager the $S_{R}$ for the GSM vortex beams, and as the spatial correlation length $\sigma_{0}$ increases, the $S_{R}$ for the GSM vortex beams first increases then decreases. The work will provide theoretical foundation for the practical application of GSM vortex beams.
\end{abstract}

\section{Introduction}

The propagation characteristics of laser beams are widely used in laser communications, remote sensing, aerial mapping and optical radar, etc[1-5]. There has been increasing interest in studying the $S_{R}$ in valuing the quality of laser beams[6-9]. Aït-Ameur reported the behavior of $S_{R}$ of Gaussian laser beam in characterising a diffracted laser beam[6]. Zalvida introduced the $S_{R}$ using the Wigner distribution function[7]. Yang etc. analysed the metrics imprived by the task accounting for the phase, spatial frequency and orientation demands based on the visual $S_{R}$ [8]. Xi studied the $S_{R}$ of partially coherent sinh-Gaussian beams in the far field propagating through atmospheric turbulence[9].

Based on the extended Huygens-Fresnel principle, the analytical expressions for the $S_{R}$ of GSM vortex beams and GSM non-vortex beams propagating through atmospheric turbulence and free space are derived, and used to study the influence of atmospheric turbulence parameters (the structure constant $C_{n}^{2}$, the inner scale $l_{0}$, the outer scale turbulence $L_{0}$ ), beam parameters (the spatial correlation length $\sigma_{0}$, the wave length $\lambda$ ).

\section{Theoretical formulation}

The field of Gaussian vortex beam at the plane $\mathrm{z}=0$ is written as [10]

$$
U(s, z=0)=\left[s_{x}+\operatorname{isgn}(m) s_{y}\right]^{|m|} \exp \left(-\frac{s_{x}^{2}+s_{y}^{2}}{w_{0}^{2}}\right),
$$

where $\operatorname{sgn}(\cdot)$ specifies the sign function, $s \equiv\left(s_{x}, s_{y}\right)$ denotes the two-dimensional position vector at the $\mathrm{z}=0$ plane, $w_{0}$ is the waist width in the Gaussian part, $m$ is the topological charge, set as \pm 1 .

By introducing the Schell correlator, the cross-spectral density function at the source plane $\mathrm{z}=0$ is expressed as[10]

$$
\begin{aligned}
W^{(0)}\left(s_{1}, s_{2}, z=0\right)= & {\left[s_{1 x} s_{2 x}+s_{1 y} s_{2 y}+\mathrm{i} \operatorname{sgn}(m) s_{1 x} s_{2 y}-\mathrm{i} \operatorname{sgn}(m) s_{2 x} s_{1 y}\right]^{|m|} } \\
& \times \exp \left(-\frac{\boldsymbol{s}_{1}^{2}+\boldsymbol{s}_{2}^{2}}{w_{0}^{2}}\right) \exp \left[-\frac{\left(\boldsymbol{s}_{1}-s_{2}\right)^{2}}{2 \sigma_{0}^{2}}\right],
\end{aligned}
$$

where $\sigma_{0}$ is the spatial correlation length, $s_{i} \equiv\left(s_{i x}, s_{i y}\right)(i=1,2)$.

By using the paraxial form of the extended Huygens-Fresnel principle, the cross-spectral density of GSM vortex beam propagating through atmospheric turbulence is expressed as[2] 


$$
\begin{aligned}
W\left(\boldsymbol{\rho}, \boldsymbol{\rho}_{d}, z\right)= & \left(\frac{k}{2 \pi z}\right)^{2} \int_{-\infty}^{\infty} \int_{-\infty}^{\infty} \int_{-\infty}^{\infty} \int_{-\infty}^{\infty} W^{(0)}\left(s, \boldsymbol{s}_{d}, z=0\right) \\
& \times \exp \left[\frac{i k}{z}(\boldsymbol{\rho}-\boldsymbol{s})\left(\boldsymbol{\rho}_{d}-\boldsymbol{s}_{d}\right)-H\left(\boldsymbol{\rho}_{d}, \boldsymbol{s}_{d}, z\right)\right] d^{2} s d^{2} \boldsymbol{s}_{d}
\end{aligned}
$$

where $k$ specifies the wavenumber related to the wave length $\lambda$ by $k=2 \pi / \lambda$. In Eq.(3) we have used the following sum and difference vector notation.

$$
\boldsymbol{\rho}=\frac{\boldsymbol{\rho}_{1}+\boldsymbol{\rho}_{2}}{2}, \boldsymbol{\rho}_{d}=\boldsymbol{\rho}_{1}-\boldsymbol{\rho}_{2}, \boldsymbol{s}=\frac{\boldsymbol{s}_{1}+\boldsymbol{s}_{2}}{2}, \boldsymbol{s}_{d}=\boldsymbol{s}_{1}-\boldsymbol{s}_{2},
$$

where the $\rho_{1}$ and $\rho_{2}$ are two arbitrary points in the receiver plane, perpendicular to the direction of propagation of the beam. In Eq.(3) the term $H\left(\boldsymbol{\rho}_{d}, \boldsymbol{s}_{d}, z\right)$ can be written as[2, 11, 12]

$$
H\left(\rho_{d}, s_{d}, z\right)=4 \pi^{2} k^{2} z \int_{0}^{1} d \xi \int_{0}^{\infty}\left[1-J_{0}\left(\kappa\left|s_{d} \xi+(1-\xi) \rho_{d}\right|\right)\right] \Phi_{n}(\kappa) \kappa d \kappa
$$

The Sterhl ratio $\left(\mathrm{S}_{\mathrm{R}}\right)$ is defined as[9]

$$
S_{\mathrm{R}}=\frac{I_{\max }}{I_{0 \max }},
$$

where $I_{\max }$ and $I_{0 \max }$ are the maximum intensity of a real beam and a diffraction-limited ideal beam, respectively. In this paper, the GSM non-vortex beam in free space is taken as the diffraction-limited ideal beam.

Letting $\rho_{1}=\rho_{2}=\rho$, the average intensity of GSM vortex beams in atmospheric turbulence obtained from Eq.(3) is written as

$$
I(\boldsymbol{\rho}, z)=W(\boldsymbol{\rho}, \boldsymbol{\rho}, z)=\frac{k^{2} w_{0}^{4}}{4\left(8 z^{2} A+k^{2} w_{0}^{2}\right)}\left[2-B\left(1-B \boldsymbol{\rho}^{2}\right) w_{0}^{2}-C\left(1-B \boldsymbol{\rho}^{2}\right)\right] \exp \left[-B \boldsymbol{\rho}^{2}\right],
$$

where

$$
\begin{aligned}
& A=\frac{1}{2 w_{0}^{2}}+\frac{1}{2 \sigma_{0}^{2}}+T, \\
& B=\frac{2 k^{2}}{8 z^{2} A+k^{2} w_{0}^{2}}, \\
& C=\frac{8 z^{2}}{\left(8 z^{2} A+k^{2} w_{0}^{2}\right) w_{0}^{2}},
\end{aligned}
$$

where

$$
T=\frac{\pi^{2} k^{2} z}{3} \int_{0}^{\infty} \kappa^{3} \Phi_{n}(\kappa) d \kappa,
$$

with $\Phi_{n}(\kappa)$ being the spatial power spectrum of the refractive index fluctuations of the turbulence, and we use the power spectrum[2]

$$
\Phi_{n}(\kappa)=0.033 C_{n}^{2} \frac{\exp \left[-\left(\kappa^{2} / \kappa_{m}^{2}\right)\right]}{\left(\kappa^{2}+\kappa_{0}^{2}\right)^{11 / 6}}(0 \leq \kappa \leq \infty),
$$

to model the Von Karman atmospheric turbulence, where $\kappa_{0}=1 / L_{0}\left(L_{0}\right.$-outer scale of the atmospheric turbulence), $\kappa_{m}=5.92 / l_{0}\left(l_{0}\right.$-inner scale of the atmospheric turbulence), $C_{n}^{2}$ is the structure constant.

Substituting Eqs.(7) and (8) into Eq.(6), we obtain

$$
S_{\mathrm{R}}=\frac{\left(8 z^{2} A_{1}+k^{2} w_{0}^{2}\right)\left[2-B w_{0}^{2}\left(1-B \boldsymbol{\rho}^{2}\right)-C\left(1-B \boldsymbol{\rho}^{2}\right)\right] \exp \left[-B \boldsymbol{\rho}^{2}\right]}{\left(8 z^{2} A+k^{2} w_{0}^{2}\right)\left[2-B_{1} w_{0}^{2}\left(1-B_{1} \boldsymbol{\rho}^{2}\right)-C_{1}\left(1-B_{1} \boldsymbol{\rho}^{2}\right)\right] \exp \left[-B_{1} \boldsymbol{\rho}^{2}\right]},
$$

For $T=0$, we get the expressions of $A_{1} 、 B_{1} 、 C_{1}$ from that of $A 、 B 、 C$. Eq.(11) is the propagation expression of $S_{R}$ of GSM vortex beam in atmospheric turbulence.

Letting $\rho_{1}=\rho_{2}=\rho, m=0$, the average intensity of GSM non-vortex beams in atmospheric turbulence obtained from Eq. (3) is written as 


$$
S_{\mathrm{R}}=\frac{\left(8 z^{2} A_{1}+k^{2} w_{0}^{2}\right) \exp \left[-B \boldsymbol{\rho}^{2}\right]}{\left(8 z^{2} A+k^{2} w_{0}^{2}\right) \exp \left[-B_{1} \boldsymbol{\rho}^{2}\right]}
$$

The values of $A, B, A_{1}, B_{1}$ are the same as in Eq. (11).

\section{Numerical calculations and analyses}

Figure1 gives the $S_{\mathrm{R}}$ of GSM vortex beams and GSM non-vortex beams propagating through atmospheric turbulence versus (a) structure constant $C_{n}^{2}$, (b) inner scale of the atmospheric turbulence $l_{0}$, and (c) outer scale of the atmospheric turbulence $L_{0}$. The calculation parameters are (a) $\sigma_{0}=1 \mathrm{~cm}$, $w_{0}=3 \mathrm{~cm}, \lambda=1064 \mathrm{~nm}, L_{0}=10 \mathrm{~m}, l_{0}=1 \mathrm{~cm}$, (b) and (c), $C_{n}^{2}=10^{-14} \mathrm{~m}^{-2 / 3}$, the other calculation parameters are the same as in Fig.1 (a). From Fig.1 (a),it can be seen that the $S_{\mathrm{R}}$ of GSM vortex beams and GSM non-vortex beams decrease with increasing structure constant $C_{n}^{2}$. From Fig.1 (b), it can be seen that the $S_{\mathrm{R}}$ of GSM vortex beams and GSM non-vortex beams increase with increasing inner scale of the atmospheric turbulence $l_{0}$. From Fig.1 (c), it can be seen that the $S_{\mathrm{R}}$ of GSM vortex beams and GSM non-vortex beams decrease with increasing outer scale of the atmospheric turbulence $L_{0}$.It can be seen from Figs.1, the $S_{\mathrm{R}}$ of GSM vortex beams propagating through atmospheric turbulence is larger than that of GSM non-vortex beams.
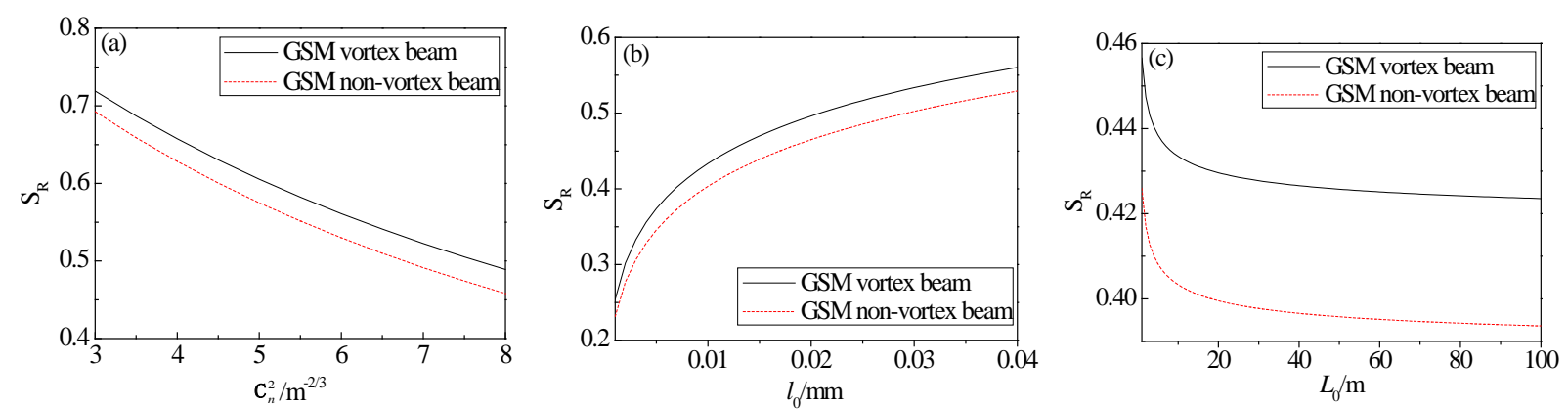

Figure 1 The $S_{\mathrm{R}}$ of GSM vortex beams and GSM non-vortex beams versus structure constant $C_{n}^{2}(\mathrm{a})$, inner scale of the atmospheric turbulence $l_{0}$ (b) and outer scale of the atmospheric turbulence $L_{0}$ (c)

The $S_{\mathrm{R}}$ of GSM vortex beams and GSM non-vortex beams propagating through atmospheric turbulence versus (a) spatial correlation length $\sigma_{0}$ and (b) wave length $\lambda$ are depicted in Figure.2(a)-2(b), respectively. The calculation parameters are $C_{n}^{2}=10^{-14} \mathrm{~m}^{-2 / 3}$, the other calculation parameters are the same as in Fig1 (a). From Fig.2(a) we see that, when $\sigma_{0}<0.028 \mathrm{~cm}$, the $S_{\mathrm{R}}$ decrease with increasing spatial correlation length $\sigma_{0}$, when $\sigma_{0}>0.028 \mathrm{~cm}$, the $S_{\mathrm{R}}$ increase with decreasing spatial correlation length $\sigma_{0}$. From Fig.2(b) we see that the $S_{\mathrm{R}}$ increase with increasing wave length $\lambda$. It can be seen from Figs.2, the $S_{\mathrm{R}}$ of GSM vortex beams propagating through atmospheric turbulence is larger than that of GSM non-vortex beams.
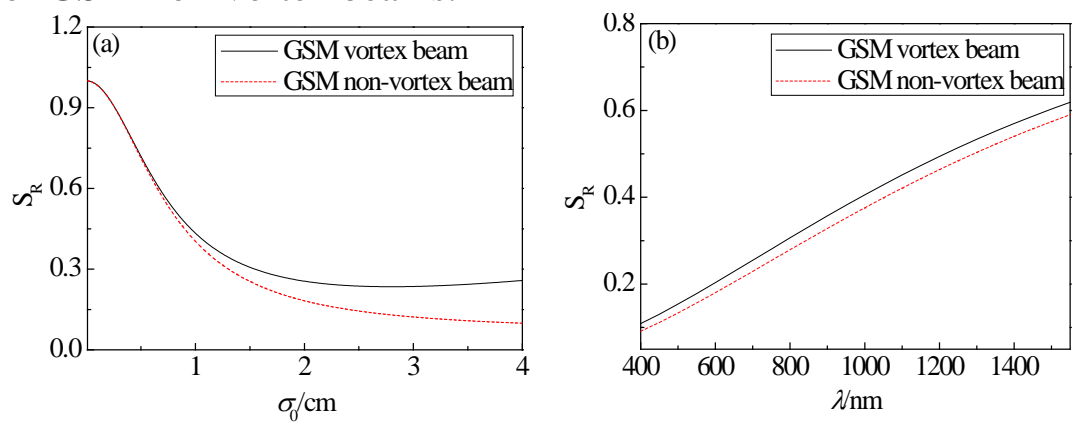

Figure 2 The $S_{\mathrm{R}}$ of GSM vortex beams and GSM non-vortex beams versus spatial correlation length $\sigma_{0}$ (a), wave length $\lambda$ (b)

\section{Conclusion}

In this paper, by using the extended Huygens-Fresnel principle, the analytical expressions for the $S_{R}$ of GSM vortex beams and GSM non-vortex beams propagating through atmospheric turbulence and 
free space are derived, and used to study the influence of beam parameters on the $S_{R}$. It is shown that with the decrement of structure constant $C_{n}^{2}$, the inner scale $l_{0}$ and the wave length $\lambda$, the increment of the outer scale turbulence $L_{0}$, the $S_{R}$ for the $S_{R}$ of GSM vortex beams and Gaussian Schell-model non-vortex beams will increase, with the increment of the spatial correlation length $\sigma_{0}$, the $S_{R}$ for the $\mathrm{S}_{\mathrm{R}}$ of GSM vortex beams and GSM non-vortex beams first decrease then increase. The theoretical analysis presented in this paper can also be used for the practical application of GSM vortex beams.

\section{Acknowledgements}

This work was financially supported by the National Natural Science Foundation of China (Nos. 61405136 and 61178067), the Natural Science Foundation for Young Scientists of Shanxi Province (Nos.2012021016 and 2013021010-4).

\section{References}

[1] Strohbehn J W: Laser beam propagation in the atmosphere (Springer-Verlag,New York,1978).

[2] Andrews L C and Phillips R L: Laser beam propagation through random media (SPIE press,Bellingham, Washington 2005).

[3] Li Jinhong and Lü Baida Acta Physics Sinica, Vol. 60 (2011) p.074205 (in Chinese)

[4] Zheng Yulong and Ji Xiaoling: High Power Laser and Particle Beams, Vol.24 (2012) p.276-280 (in Chinese)

[5] Duan Meiling,Li Jinhong and Wei Jinlin: High Power Laser and Particle, Vol.25 (2010) p.2252-2256 (in Chinese)

[6] Aït-Ameur K: Optics Communications Vol. 285 (2012), p. 699

[7] Zalvidea D, Lehman M and Granieri S,Sicre E E: Optics Communications Vol. 118 (1995), p. 207

[8] Yang Y, Cao G and Zhao H: Optik - International Journal for Light and Electron Optics Vol. 124 (2013), p. 6415

[9] Xiao X, Ji X and Lü B: Optics \& Laser Technology Vol. 40 (2008), p. 129

[10] Li J, Zhang H and Lü B: Optics \& Laser Technology Vol. 42 (2010), p. 428

[11] Dan Y and Zhang B: Opt. Express Vol. 16 (2008), p. 15563

[12] Yuan Y, Cai Y, Eyyuboğlu H T and Baykal Y,Chen J: Optics and Lasers in Engineering Vol. 50 (2012), p. 752 